Research in Astronomy and Astrophysics manuscript no.

(LTEX: Cuixh'Nov12.tex; printed on August 22, 2018; 14:41)

\title{
Delayed Onset and Fast Rise of Prompt Optical-UV Emission from Gamma-Ray Bursts in Molecular Clouds
}

\author{
Xiao-Hong Cui ${ }^{1}$ Zhuo $\mathrm{Li}^{2,3}$ and Li-Ping $\mathrm{Xin}^{1}$ \\ 1 National Astronomical Observatories, Chinese Academy of Sciences, Beijing 100012, China; xhcui@bao.ac.cn \\ 2 Department of Astronomy, Peking University, Beijing 100871, China \\ ${ }^{3}$ Kavli Institute for Astronomy and Astrophysics, Peking University, Beijing 100871, China
}

\begin{abstract}
Observations imply that long $\gamma$-ray bursts (GRBs) are originated from explosions of massive stars, therefore they may occur in the molecular clouds where their progenitors were born. We show here that the prompt optical-UV emission from GRBs may be delayed due to the dust extinction, which can well explain the observed optical delayed onset and fast rise in GRB 080319B. The density and the size of the molecular cloud around GRB 080319B are roughly constrained to be $\sim 10^{3} \mathrm{~cm}^{-3}$ and $\sim 8$ pc, respectively. We also investigate the other GRBs with prompt optical-UV data, and find similar values of the densities and sizes of the local molecular clouds. The future observations of prompt optical-UV emission from GRBs in subsecond timescale, e.g., by UFFO-Pathfinder and SVOM-GWAC, will provide more evidence and probes of the local GRB environments.
\end{abstract}

Key words: radiation mechanisms: non-thermal - gamma-rays: bursts - dust: extinction

\section{INTRODUCTION}

The properties of $\gamma$-ray burst (GRB) circumburst and host-galaxy environment are important for the studies of GRB progenitors and the fundamental conditions required within a galaxy to form a GRB. The multi-wavelength observations about the emission from GRBs and that from their host galaxies would provide a unique tool to understand the nature of GRBs and the properties of interstellar medium (ISM) around the bursts.

Observations imply that long GRBs are originated from massive star explosions. First, they are observed to lie in starforming galaxies, or even within the active star-forming regions of the host galaxies (e.g., Paczyński 1998; Bloom, Djorgovski, \& Kulkarni 2002). More precise HST images of afterglows reveals that they occur within a few kiloparsecs of the fluxweighted centroid of their host galaxies (Fruchter et al. 2006). Second, X-ray observations show evidence for high column densities of gas around long GRBs, implying giant molecular clouds around them (e.g., Galama \& Wijers 2001). Finally, at least some long GRBs are associated with core-collapse supernovae ( $\mathrm{SNe}$ ). The discovery of four clear associations between long, soft GRBs and Type Ib/c SNe and many SN-like bumps in the late optical afterglow light curves (see, e.g., review by Woosley \& Bloom 2006) directly tell that their progenitors are massive stars. 
Now that the progenitors of long GRBs are massive stars, they may occur in the birth place of the progenitors since massive stars are short-lived, i.e., the long GRBs may lie in the molecular clouds that the massive stars are born. The optical-UV and Xray emission from GRBs can be affected significantly by the extinction of dust and absorption of gas in the local environment, $\gamma$-ray emission is almost unaffected. Therefore, one may naturally expect that the behavior of prompt optical-UV emission is different in light curves from that of prompt $\gamma$-ray emission. The difference may hint the properties of the dust environments around the GRBs. The interaction of a GRB with the environment can yield powerful clues on the properties of the medium in which the burst occur. The behavior of the X-ray and optical opacities in the nearby of a GRB have been studied (Perna, Raymond, \& Loeb 2000; Perna\& Raymond 2000). A time-dependent photoionization code has been developed to study the modificaitons in the dust distribution and the graphite in the medium around the GRB was found to be more resistent than silicates (Perna \& Lazzati 2002; Lazzati \& Perna 2002).

The varieties of observed GRB prompt optical behaviors are rich. The prompt optical emission was first observed in GRB 990123 and was found to be uncorrelated with the ongoing $\gamma$-ray emission (Akerlof et al. 1999; but see Liang et al. 1999). Then the prompt optical emission from GRB 050820A (Vestrand et al. 2006) was reported and a strong correlation between $\gamma$-energy and optical emission in the prompt phase was discovered. Similar cases of some degree of correlation are observed in GRB 041219A (Vestrand et al. 2005; Blake et al. 2005), GRB 060526 (Thöne et al. 2010), and “naked eye” burst GRB 080319B (Racusin et al. 2008; Beskin et al. 2010). GRB 080319B with the richest prompt optical observation attracted much attention about it's nature. The detailed observation of this burst presented by Racusin et al. (2008) showed not only a correlation between $\gamma$-ray and optical emission in the prompt phase but also an obvious delayed onset $\sim 15 \mathrm{~s}$ between them.

In this work, we show that if a GRB is located in a molecular cloud, its prompt optical-UV emission may be absorbed by the dust in the molecular cloud, and only emerges after the dusts on the line of sight are all destroyed. This can well explain the observed delayed onset of the prompt optical-UV emission in GRB 080319B, and the density and the size of the molecular cloud around this burst can be roughly constrained. For other bursts with prompt optical observations, the properties of local environment can also be constrained. We find similar properties of the clouds, with density and size being $n_{\mathrm{H}} \sim 10^{3}-10^{4}$ $\mathrm{cm}^{-3}$ and $\Delta R \sim 6 \mathrm{pc}$. The paper is arranged as the following: a simple model of the radiation-dust interaction and the resulted prompt optical-UV light curve are presented in Section 2; in Section 3, we apply the model to GRB 080319B and other GRBs with prompt optical-UV observations; in Section 4 discussions and conclusion are presented.

\section{RADIATION-DUST INTERACTION AND EMERGENT OPTICAL-UV EMISSION}

Consider a GRB that is located in a molecular cloud. The prompt optical-UV emission from this GRB may be absorbed by the dust in the cloud, but in the same time the dusts may also be destroyed by the emission. If the optical-UV emission is strong and lasts long enough, it may emerge from the cloud after the dusts on the way are all destroyed. The dust destruction by the optical-UV radiation has been discussed by Waxman \& Draine (2000). Here we will follow their model in the radiation-dust interaction, and focus on the back effect of the dust on the optical-UV emission, i.e., how the dusts in a cloud of finite size affects the apparent light curve of the prompt optical-UV emission. On the other hand, from the observed light curve profile of the prompt optical-UV emission, we can also give some constraints on the properties of the molecular cloud. We will only consider dust destruction due to thermal sublimation and neglect the effect of grain charging, since, as argued by Waxman \& Draine (2000) and Draine \& Hao (2002), the thermal sublimation is likely to be more effective (see, but, Fruchter et al. 2001). 


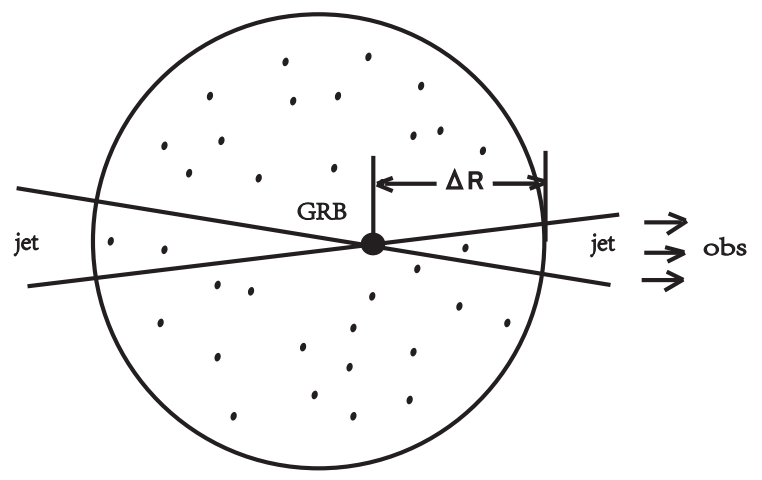

Fig. 1 The sketch of an observed GRB located inside a molecular cloud. "obs" denotes the direction to the Earth (observer). The cloud is assumed to be with uniform density and a clear boundary. The distance of the GRB to the edge of the cloud is $\Delta R$, as marked.

Considering a simple picture as shown in Fig 1, the molecular cloud is assumed to be uniform in density, and the distance of the GRB from the edge of the cloud on the side to the observer is $\Delta R$. The cloud contains dust grains of characteristic radius $a$ and dust number density $n_{d}$. Assuming a standard dust-to-gas mass ratio, $n_{d}$ is related to the cloud density $n_{\mathrm{H}}$ as $n_{d}=0.01 n_{\mathrm{H}} m_{\mathrm{H}} /(4 \pi / 3) a^{3} \rho$, where $\rho$ is mass density of the grain material. A characteristic value of $\rho=3.5 \mathrm{~g} \mathrm{~cm}^{3}$ (Guhathakurta \& Draine 1989) will be taken in the following calculations. Considering that the source radiates a $1-7.5 \mathrm{eV}$ radiation with luminosity $L_{1-7.5}$, a grain at a distance $r$ can be heated up leading to thermal sublimation and thermal emission. The temperature $T$ of the grain at distance $r$ from the source is governed by

$$
\frac{L_{1-7.5}}{4 \pi r^{2}} Q \mathrm{UV} \pi a^{2}=\langle Q\rangle_{T} 4 \pi a^{2} \sigma T^{4}-4 \pi a^{2} \frac{d a}{d t} \frac{\rho}{m} B,
$$

where $m$ is the mean atomic mass, $B$ is the chemical binding energy per atom, $Q_{\mathrm{UV}}$ is the absorption efficiency factor averaged over the $1-7.5 \mathrm{eV}$ spectrum of the source emission, and $\langle Q\rangle_{T}$ is the usual Planck-averaged absorption efficiency. We will assume $Q_{\mathrm{UV}} \approx 1$ for $a \gtrsim 10^{-5} \mathrm{~cm}$ and approximate $\langle Q\rangle_{T}$ by

$$
\langle Q\rangle_{T} \approx \frac{0.1 a_{-5}(T / 2300 \mathrm{~K})}{1+0.1 a_{-5}(T / 2003 \mathrm{~K})}
$$

with $a_{-5}=a / 10^{-5} \mathrm{~cm}$. The thermal sublimation rate can be approximated by (Guhathakurta \& Draine 1989)

$$
\frac{d a}{d t}=-\left(\frac{m}{\rho}\right)^{1 / 3} \nu_{0} e^{-B / k T} .
$$

We adopt the frequency $\nu_{0}=1 \times 10^{15} \mathrm{~s}^{-1}, B / k=7 \times 10^{4} \mathrm{~K}$, and $\rho / \mathrm{m}=10^{23} \mathrm{~cm}^{-3}$ as representative values (Guhathakurta \& Draine 1989; Waxman \& Draine 2000). If we assume $T$ is approximately constant during the illumination, then the grain survival time at $T$ is $t_{\text {surv }}(T)=a /|d a / d t|$. The grain will be completely destructed by thermal sublimation if it is illuminated over a time longer than $t_{\text {surv }}(T)$.

As the dust is destructed by the radiation, the radiation is also extinguished by the dust. We consider below the effects of dust extinction on the observed flash light curves. Following Waxman \& Draine (2000) let us approximate the $1-7.5$ 
$\mathrm{eV}$ emission from the GRB as a rectangular pulse of duration $\Delta t$ and luminosity $L_{1-7.5}$. The problem can be simplified by assuming that the effects of extinction can be approximated as a narrowing of the optical pulse, retaining a rectangular profile. The leading edge of the radiation is just at the dust destruction front. We assume a sharp disruption front within which the dust grains are all destructed whereas the grains further are not affected. Define $f(r)$ the fraction of the flash energy that is absorbed by dust interior to radius $r$. If $t_{\text {surv }}<(1-f) \Delta t$, the grains are destructed and $f(r)$ satisfies

$$
\frac{d f}{d r}=Q_{\mathrm{UV}} n_{d} \pi a^{2} \frac{t_{\mathrm{surv}}}{\Delta t} .
$$

The relation between the radius of dust destruction front $R_{f}$ and observer time $t_{\mathrm{obs}}$ can be given by

$$
t_{\mathrm{obs}}=f\left(R_{f}\right) \Delta t(1+z),
$$

with $z$ the redshift of the GRB source. A (maximum) dust destruction radius $R_{d}$ is determined by the condition $t_{\text {surv }}\left[T\left(R_{d}\right)\right]=$ $\left[1-f\left(R_{d}\right)\right] \Delta t$. At $r>R_{d}$, the dust grain survives the illumination and the destruction front does not move any more, therefore we can simply assume $R_{f}=R_{d}$ and $f=1$ at $t_{\mathrm{obs}}>f\left(R_{d}\right) \Delta t(1+z)$.

The above discussion on $R_{f}$ propagation omits the existence of the edge of the cloud at $\Delta R$. If $R_{f}<\Delta R$, the optical depth due to dust extinction is

$$
\tau=Q_{\mathrm{UV}} n_{d} \pi a^{2}\left(\Delta R-R_{f}\right),
$$

and the attenuated luminosity observed outside is

$$
L_{\text {obs }}\left(t_{\text {obs }}\right)=L_{1-7.5} \exp \left\{-\tau\left[R_{f}\left(t_{\text {obs }}\right)\right]\right\} \text {. }
$$

If $R_{d}>\Delta R$ then the destruction front can reach the edge $\left(R_{f}=\Delta R\right)$ at time $t_{\mathrm{obs}}=f(\Delta R) \Delta t(1+z)$, which means all the dust in the beam of the radiation is cleared and the radiation is not attenuated, $\tau=0$. However, if $R_{d}<\Delta R$ then the dust is not destructed completely, and the disruption front stays at $R_{d}$ at $t_{\mathrm{obs}}>f\left(R_{d}\right) \Delta t(1+z)$ while the dust optical depth is fixed at $\tau=Q_{\mathrm{Uv}} n_{d} \pi a^{2}\left(\Delta R-R_{d}\right)$.

Note, in the former case $R_{d}>\Delta R$, the $1-7.5 \mathrm{eV}$ emission is firstly totally attenuated, since $R_{f}<\Delta R$ and $\tau \gg 1$; when the destruction front propagates to be close to the edge of the cloud, $R_{f} \lesssim \Delta R$ and $\tau \sim 1$, it starts to emerge by some fraction; and after the destruction front reaches the edge, $R_{f}=\Delta R$, the emission emerge completely without any extinction. Thus, the end time of the light curve rising up depends on the edge of cloud $\Delta R$, while the slope of the light curve rising up depends on the propagation speed of the destruction front which is sensitive to the cloud density, $n_{\mathrm{H}}$.

\section{APPLICATIONS}

As discussed above, in the case of $R_{d}>\Delta R$, the radiation-dust interaction leads to that only the later part of the prompt optical-UV photons emerges, but the $\gamma$-ray photons from the GRB are without any attenuation. Thus, if the prompt $\gamma$-ray and optical-UV radiation is emitted together from the GRB source, there should be a time delay between the onset of the apparent prompt optical-UV and $\gamma$-ray emission. So far there are quite a few GRBs that are detected with prompt optical-UV emission. We will apply the simple radiation-dust interaction model to all these detected GRBs, with the goal to explain the time delays of the prompt optical-UV emission relative to $\gamma$-ray emission, and roughly give some implications to the properties, e.g., the densities and the sizes of the molecular clouds around them. 
The observed luminosity is usually given in a single band for a single filter, e.g., U, B, V, R bands etc. A spectrum with the form $f_{\nu} \propto \nu^{-1}$ is assumed for the prompt optical-UV flash in 1-7.5 eV, which is consistent with the fast-cooling electrons expected in the standard internal shock model. Thus, a cosmological $\kappa$-correction factor can be defined to account for the transformation of the single passband of filter to the band of $1-7.5 \mathrm{eV}$ in the proper GRB frame,

$$
\kappa=\frac{\int_{1 \mathrm{eV} / h(1+z)}^{7.5 \mathrm{eV} / h(1+z)} f_{\nu} d \nu}{\int_{b_{1}}^{b_{2}} f_{\nu} d \nu}
$$

where $b_{2}$ and $b_{1}$ are the frequency boundaries of the passband for the observed filter, $z$ is the GRB redshift.

\subsection{GRB 080319B}

So far the so-called "naked-eye" GRB 080319B is the only one that happens to occur in the field of view of an optical telescope, without the trigger by high-energy detector, thus it is by luck monitored in optical band from before the beginning of the GRB. The broadband observations of it has been presented by Racusin et al. (2008) and Beskin et al. (2010). The $\gamma$-ray emission was found to begin at about $4 \mathrm{~s}$ before the BAT trigger and last $\sim 57 \mathrm{~s}$. The bright optical transient begins at $\sim 10 \mathrm{~s}$ after the BAT trigger, peaks at $\sim 18 \mathrm{~s}$ and then fades below the threshold to magnitude $\sim 12$ after $5 \mathrm{~min}$. That is to say, there is a time delay $\sim 14 \mathrm{~s}$ between the onsets of $\gamma$-ray and optical emission. It should be noticed that the optical rising is too fast to be accounted for by the afterglow model, either forward shock emission (Sari et al. 1998) or reverse shock emission (Kobayashi et al. 2000). The optical light curve during the plateau phase shows fluctuation, similar to the $\gamma$-ray one. Moreover, the optical and $\gamma$-ray emission is found to be correlated. All these features suggest that the prompt optical emission from this burst is not produced by afterglow shock. Thus the delayed up-rising optical emission needs other explanation. We show below that the delay can be well explained by the radiation-dust interaction.

We, again, approximate the intrinsic optical-UV emission as a rectangle pulse of duration $\Delta t$ (in the rest frame of the $\mathrm{GRB}$ ). Since the optical emission is observed to decay at $\approx 50$ s (similar to the $\gamma$-ray duration), the duration is $\Delta t \approx 50 /(1+$ $z) \approx 25 \mathrm{~s}$, where the GRB redshift is $z=0.937$ (Vreeswijk et al. 2008). Apparently in observations, the optical flux rises from zero to a plateau phase at a time $t_{b} \approx 15 \mathrm{~s}$ after trigger, and the plateau phase ends at $\sim 50 \mathrm{~s}$. The mean luminosity after $t_{b}$ (i.e.,in the range of $15-50 \mathrm{~s}$ ) and in 1-7.5 eV energy band can be given by $L_{1-7.5}=4 \pi D_{L}(z)^{2} \kappa f_{p} \approx 2.6 \times 10^{50} \mathrm{erg}$ $\mathrm{s}^{-1}$. Here $D_{L}(z)$ is the luminosity distance calculated (adopting a Universe model with $\Omega_{\mathrm{M}}=0.3, \Omega_{\Lambda}=0.7$, and $H_{0}=71$

$\mathrm{km} \mathrm{s}^{-1} \mathrm{Mpc}^{-1}$ ), and $f_{p}=9.39 \times 10^{-9} \mathrm{erg} \mathrm{cm}^{-} 2 \mathrm{~s}^{-1}$ is the mean flux observed during the time range $15-50 \mathrm{~s}$ in $\mathrm{V}$ band observed by TORTORA (Pagani et al. 2008; Racusin et al. 2008). The correction factor $\kappa=6.17$ for this burst is calculated by assuming a power law spectrum $f_{\nu} \sim \nu^{-1}$.

If the duration of the optical-UV emission $\Delta t=25 \mathrm{~s}$ is taken and assuming the radius of dust grain as $a=1 \times 10^{-5}$ $\mathrm{cm}$, we calculate the absorbed energy fraction $f(r)$ of the flash up to the destruction radius $R_{d}$ for a cloud density range of $n_{\mathrm{H}}=10^{2}-10^{5} \mathrm{~cm}^{-3}$. The result is shown in Fig 2 We can see the denser the cloud the faster the flash energy is absorbed. However, after the destruction front reaches the destruction radius $r=R_{d}$, the absorbed fraction rapidly reaches unity, $f\left(R_{d}\right)=1$.

Apparently in observations, the optical emission rises to the mean flux level at about $15 \mathrm{~s}$, and then keeps this level until $\sim 50 \mathrm{~s}$. This implies that the absorbed fraction of the flash energy, when the dust destruction front reaches the edge of the cloud, is $f\left(R_{f}=\Delta R\right) \approx 15 / 50=0.3$. Thus, given the cloud density $n_{\mathrm{H}}$, the cloud size, roughly implicated by $\Delta R$, can be determined for this burst, i.e. $n_{\mathrm{H}}$ and $\Delta R$ are one by one related for fixed $f(r=\Delta R)$ value. For example, if 


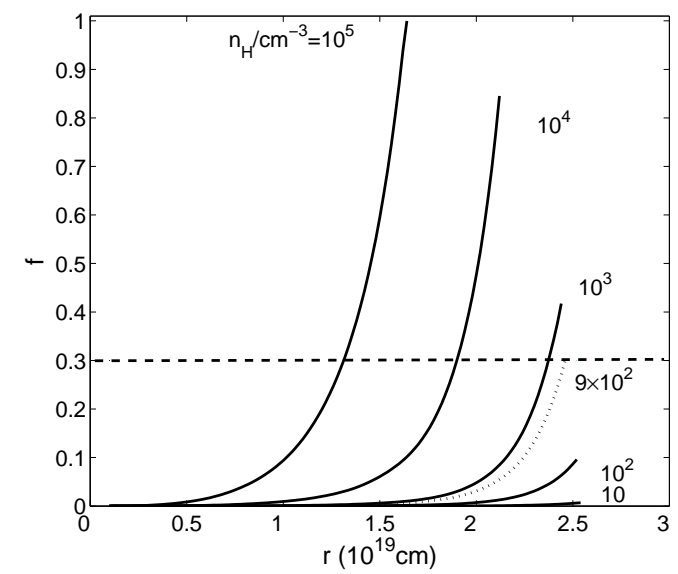

Fig. 2 The fraction $f(r)$ of flash energy absorbed by dust interior to radius $r$ up to dust destruction radius $r=R_{d}$ in the case of GRB 080319B. The duration and luminosity of prompt emission in $1-7.5 \mathrm{eV}$ are $\Delta t=25 \mathrm{~s}$ and $L_{1-7.5}=2.6 \times 10^{50} \mathrm{erg} \mathrm{s}^{-1}$, respectively, and the dust grain size is assumed to be $a=10^{-5} \mathrm{~cm}$. Different lines correspond to different values of cloud density $n_{\mathrm{H}}$, as marked in the plot. The dash line shows $f(r=\Delta R)=0.3$. The dotted line presents the case of $f\left(r=R_{d}\right)=0.3$, i.e., $R_{d}=\Delta R$.

$n_{\mathrm{H}}=\left(10^{3}, 10^{4}, 10^{5}\right) \mathrm{cm}^{-3}$, we have $\Delta R=(2.4,1.7,1.1) \times 10^{19} \mathrm{~cm}$, respectively with fixed $f(r=\Delta R)=0.3$. Then the value of $\Delta R$ can be found to decrease with larger value of $n_{\mathrm{H}}$. However, for too small $n_{\mathrm{H}}$, the destruction front reaches the maximum destruction radius $R_{d}$ before reaching the edge of the cloud, i.e., the absorbed fraction $f\left(r=R_{d}\right)<0.3$, as the case of $n_{\mathrm{H}}=10^{2} \mathrm{~cm}^{-3}$ in Fig 2

In order to decouple $n_{\mathrm{H}}$ and $\Delta R$, we need to further consider the temporal profile of the observed optical-UV emission. For different values of $n_{\mathrm{H}}$ and $t_{b}$, we have calculated the optical-UV light curve using equation (7). The resulted light curves are shown in Fig 3, also plotted are the $\gamma$-ray and optical-UV data that are adopted from Racusin et al. (2008). Note, as Beskin et al. (2010) found that the optical emission is $2 \mathrm{~s}$ delayed relative to the $\gamma$-ray emission in the plateau phase, we also assume a time delay of $2 \mathrm{~s}$ for the intrinsic onset of optical-UV emission compared to the $\gamma$-ray one. The plotted light curves in Fig. 3 take this into account. We see that compared with the observed optical data of GRB $080319 \mathrm{~B}$, the case with $n_{\mathrm{H}}=10^{3} \mathrm{~cm}^{-3}$ and $t_{b}=16 \mathrm{~s}$ fits the light curve profile better. Therefore, it can be concluded that the cloud that hosts GRB 080319B has a density of $n_{\mathrm{H}} \approx 10^{3} \mathrm{~cm}^{-3}$ and a size of $R \sim \Delta R \approx 8 \mathrm{pc}$.

It should be noted that in the above calculations we have taken $\rho=3.5 \mathrm{~g} \mathrm{~cm}^{3}$ (Guhathakurta \& Draine 1989), $a=$ $1 \times 10^{-5} \mathrm{~cm}$ and a standard dust-to-gas mass ratio of 0.01 . The resulted values of $n_{\mathrm{H}}, \Delta R$ and $R_{d}$ are not sensitive to the values of them, i.e., the resulted $n_{\mathrm{H}}, \Delta R$ and $R_{d}$ values vary within a factor of a few if changing $\rho, a, \kappa$, and the gas-to-dust ratio by one order of magnitude. This is good enough for order of magnitude estimate with the simple model here.

\subsection{Other GRBs with prompt optical detections}

Besides GRB 080319B, there are quite a few other GRBs with prompt optical detections during the $\gamma$-ray bursting phase. They are all detected by rapid slew of optical telescopes to the GRB location after trigger by $\gamma$-ray detectors. So usually there is a 

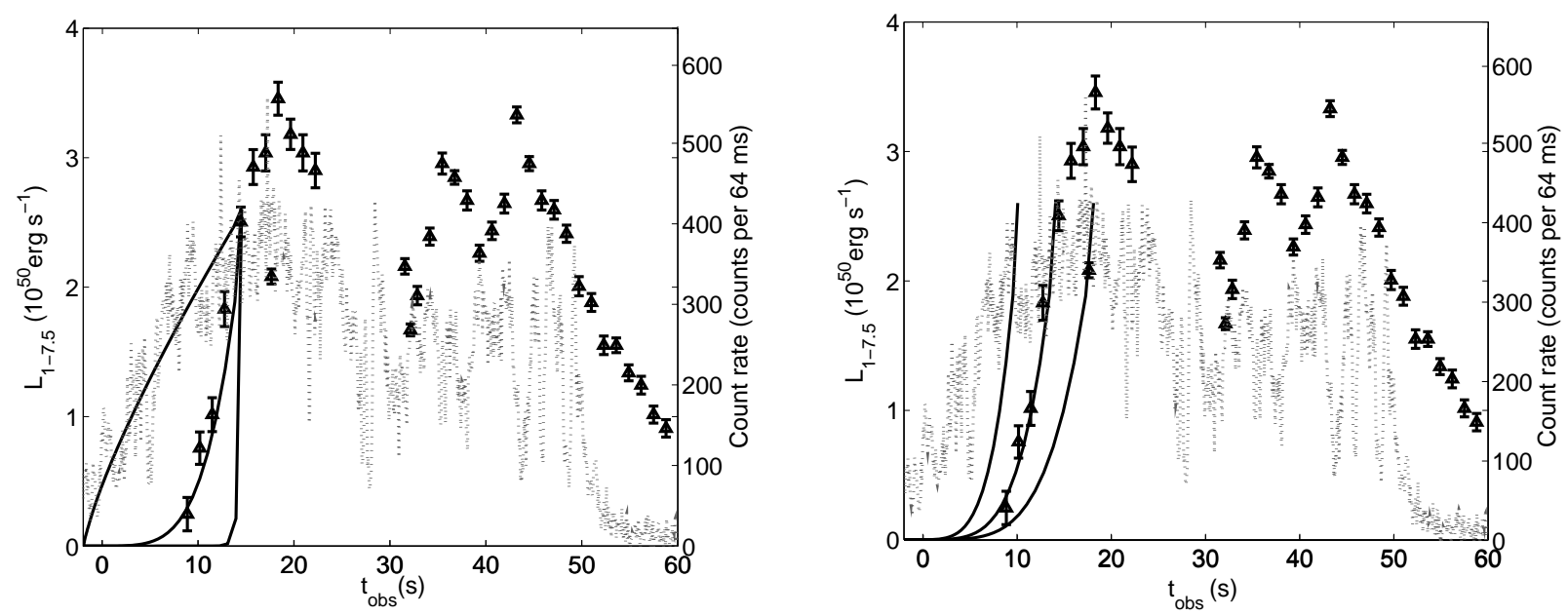

Fig. 3 The light curves of GRB 080319B in $\gamma$-ray and optical bands. The black triangles are optical data from TORTORA. For comparison, the Konus-Wind background subtracted $\gamma$-ray light curve $(18-1,160 \mathrm{keV})$, with respect with the trigger time by Swift-BAT, is shown with dashed line. The solid lines are the calculated optical light curves in the simple radiation-dust interaction model. Left panel: The cases with the cloud density $n_{\mathrm{H}}=$ $10^{2}, 10^{3}, 10^{4} \mathrm{~cm}^{-3}$ (corresponding to three light curves from left to right respectively) and the fixed end time of the rising part $t_{b}=16 \mathrm{~s}$. Right panel: The cases with $t_{b}=12,16,20 \mathrm{~s}$ (from left to right) and the fixed $n_{\mathrm{H}}=10^{3} \mathrm{~cm}^{-3}$. The other parameters are the same as Fig.2. The case with $t_{b}=16 \mathrm{~s}$ and $n_{\mathrm{H}}=10^{3} \mathrm{~cm}^{-3}$ gives the best fit to the rising part of the optical flash of GRB 080319B.

gap between the trigger time and the start time of optical observation. Nevertheless, we can still try to make some constraints on the local GRB environments based on the simple radiation-dust interaction model.

All the GRBs detected after December of 2004 and with optical detections during the prompt $\gamma$-ray emission are collected and analyzed with the simple radiation-dust interaction model here. We separate these GRBs into two samples. In Sample I, the GRBs satisfie the following three criterions: (1) There are optical detections before the end of the GRB, specifically, the optical detection is within the duration of $T_{90}$; (2) The optical light curve within $T_{90}$ shows a rising of the flux, i.e., if the optical light curve shows a decay or flat plateau then the GRB is not included; (3) The number of optical data points, excluding upper limits, in the rising part is not less than three. All the other GRBs only satisfy criterion (1) are grouped into Sample II.

We find besides GRB 080319B, there are 7 other GRBs satisfy the three criterions: GRB 041219A (Vestrand et al. 2005; Blake et al. 2005); GRB050820A (Vestrand et al. 2006); GRB 060218 (Mirabal et al. 2006; Ferrero et al. 2006; Sollerman et al. 2006; Kocevski et al. 2007); GRB 060418 (Molinari1 et al. 2007; Dupree et al. 2006; Vreeswijk \& Jaunsen 2006), GRB 060607A (Molinari1 et al. 2007; Ledoux et al. 2006), GRB 080810 (Page et al. 2009; Burenin et al. 2008), and GRB 100906A (Gorbovskoy et al. 2011; Barthelmy et al. 2010; Markwardt et al. 2010; Tanvir et al. 2010). However, we exclude GRB 041219A and GRB 060218 from Sample I for reasons as follows. GRB 041219A shows correlation between $\gamma$-ray and optical emission, thus the observed initial rising in the optical band is likely intrinsic (Vestrand et al. 2005) other than due to radiation-dust interaction. This GRB is included in Sample II instead. As for GRB 060218, its early optical-UV emission is likely associated with the supernova shock breakout (Campana et al. 2006; Waxman et al 2007), thus not due to radiation-dust 
Table 1 The observational results of GRBs in Sample I and the constraints of their local molecular clouds

\begin{tabular}{cccccccccccc}
\hline GRB & $z$ & $\begin{array}{c}T_{90} \\
(\mathrm{~s})\end{array}$ & $\begin{array}{c}t_{\mathrm{op}} \\
(\mathrm{s})\end{array}$ & $\kappa^{*}$ & $\begin{array}{c}L_{1-7.5} \\
\left(10^{48} \mathrm{erg} \mathrm{s}^{-1}\right)\end{array}$ & $\begin{array}{c}R_{\mathrm{d}} \\
(\mathrm{pc})\end{array}$ & $\begin{array}{c}\Delta R \\
(\mathrm{pc})\end{array}$ & $\begin{array}{c}n_{\mathrm{H}} \\
\left(10^{3} \mathrm{~cm}^{-3}\right)\end{array}$ & $\begin{array}{c}\Delta t_{\mathrm{obs}} \\
(\mathrm{s})\end{array}$ & $\begin{array}{c}t_{b} \\
(\mathrm{~s})\end{array}$ \\
\hline 050820A & 2.6 & $750^{* *}$ & 84 & $3.10(\mathrm{R})$ & 0.6 & 3.35 & 3.23 & 9 & 646 & 305 & $1,2,3$ \\
060418 & 1.49 & 103.1 & 40 & $4.07(\mathrm{H})$ & 2.4 & 6.91 & 6.87 & 4 & 140 & 107 & $4,5,6$ \\
060607A & 3.082 & 102 & 73 & $4.07(\mathrm{H})$ & 2.3 & 5.90 & 5.87 & 5 & 200 & 150 & 4,7 \\
080319B & 0.937 & 57 & $8.9 \dagger$ & $6.17(\mathrm{~V})$ & 260 & 7.78 & 7.67 & 1 & 50 & 16 & $8,9,10$ \\
080810 & 3.35 & 106 & 38 & $3.10(\mathrm{~W})$ & 7.1 & 11.7 & 11.4 & 3 & 150 & 67 & 11,12 \\
$100906 \mathrm{~A}$ & 1.727 & 114.4 & 48.5 & $3.10(\mathrm{~W})$ & 1.2 & 4.60 & 4.48 & 15 & 190 & 83 & $13,14,15,16$ \\
\hline
\end{tabular}

* In the bracket is the passband of filter. Letters "V", "H" and "W" denote V, H and white bands, respectively.

** From the work of Vestrand et al. (2006), rather than Swift data.

$\dagger$ The time corresponds to the first optical data by TORTORA. In fact, the optical observations start before the trigger of this GRB. References: (1) Prochaska et al. 2005; (2) Ledoux et al. 2005; (3) Vestrand et al. 2006; (4) Molinari1 et al. 2007; (5) Dupree et al. 2006; (6) Vreeswijk \& Jaunsen 2006; (7) Ledoux et al. 2006; (8) Vreeswijk et al. 2008; (9) Racusin et al. 2008; (10) Beskin et al. 2010; (11) Page et al. 2009; (12) Burenin et al. 2008; (13) Gorbovskoy et al. 2011; (14) Barthelmy et al., 2010; (15) Markwardt et al., 2010; (16) Tanvir et al. 2010

interaction either. Sample I GRBs are listed in Table 1, All the other GRBs only satisfy criterion (1) are grouped into Sample II. For example, GRB 110205A (Klotz et al. 2011a, b; Cucchiara et al. 2011) was started to be detected in optical band $166 \mathrm{~s}$ after trigger but within the duration of $T_{90}=257 \mathrm{~s}$. The observed optical light curve already appears to be a plateau, without a rising part, which may occur before the start of optical detections. We exclude this burst in Sample I but include in Sample II. As shown in Table2, there are 13 GRBs in Sample II.

\subsubsection{Sample I}

For GRBs in Sample I, we can follow the same approach we carry for GRB 080319B, and the optical rising can be accounted for by the simple radiation-dust interaction model, in the same time the local environments of these bursts are constrained by fitting the observed promptly optical-UV light curves.

We assume there is intrinsic optical-UV emission associated with the $\gamma$-ray emission, with approximated rectangle light curve profile. The optical-UV duration $\Delta t_{\mathrm{obs}}=\Delta t(1+z)$ is obtained from observations (which is usually comparable or somewhat larger than the $\gamma$-ray duration). The luminosity $L_{1-7.5}$ is calculated from observed optical emission, by correction with $\kappa$ factor assuming a $f_{\nu} \propto \nu^{-1}$ spectrum (For white band the same $\kappa$ factor as $\mathrm{R}$ band is assumed). There are usually fluctuations of optical flux in the plateau phase, thus we use the average of optical flux during the plateau phase (i.e., after the rising part and before the decay phase) to calculate the $L_{1-7.5}$ values: We average the optical data of GRB 050820A during the period of $t_{\mathrm{obs}}=230-722 \mathrm{~s}$; GRB 060418 of $107-137 \mathrm{~s}$; GRB $060607 \mathrm{~A}$ of $159-205 \mathrm{~s}$; GRB 080810 of $67-261 \mathrm{~s}$; and for GRB 100906A we use the peak flux at 115s.

Given $L_{1-7.5}$ and $\Delta t$, the maximum dust destruction radius $R_{d}$ can be determined to be as function as density $n_{\mathrm{H}}$. Furthermore, the time $t_{b}$ that the optical flux rise to the top value can be estimated from the observed optical light curve. Once given $f(r=\Delta R)=t_{b} / \Delta t_{\mathrm{obs}}$ and combined with the condition of $R_{d}=\Delta R$, one obtains a minimum $n_{\mathrm{H}}$ value, $n_{\mathrm{H}}>n_{\mathrm{H}, 0}$, otherwise, the destruction front cannot reach the edge of the cloud and no optical-UV emission escapes from the 
Table 2 The observational results of GRBs in sample II and the constraints of their local molecular clouds

\begin{tabular}{cccccc}
\hline GRB & $z$ & $\begin{array}{c}T_{90} \\
(\mathrm{~s})\end{array}$ & $\begin{array}{c}t_{\mathrm{op}} \\
(\mathrm{s})\end{array}$ & $\begin{array}{c}L_{1-7.5} \\
\left(10^{47} \mathrm{erg} \mathrm{s}^{-1}\right)\end{array}$ & ref \\
\hline $041219 \mathrm{~A}$ & 0.31 & 520 & 460 & $3.7 \times 10^{-5}$ & 1,2 \\
050319 & 3.24 & 160.5 & 30.4 & 3.9 & 3,4 \\
050904 & 6.29 & 174.2 & 150.3 & 1.1 & 5 \\
060526 & 3.21 & 298.2 & 16.1 & 3.4 & 6 \\
$060904 \mathrm{~B}$ & 0.703 & 171.5 & 21 & $<6.8 \times 10^{-2}$ & 7 \\
061126 & 1.16 & 70.8 & 42 & $1.9 \times 10^{-2}$ & 8,9 \\
071003 & 1.1 & 150 & 44.5 & 3.0 & 10 \\
071031 & 2.69 & 180 & 59.6 & 2.3 & 11,12 \\
$080603 \mathrm{~A}$ & 1.69 & 150 & 105 & $5.8 \times 10^{-3}$ & 13 \\
080607 & 3.036 & 79 & 24.5 & 2.7 & 14 \\
$100901 \mathrm{~A}$ & 1.408 & 439 & 113.4 & $9.6 \times 10^{-2}$ & $15,16,17$ \\
$100902 \mathrm{~A}$ & 4.5 & 428.8 & 104 & $<1.4$ & 17 \\
$110205 \mathrm{~A}$ & 1.98 & 257 & 166 & 0.2 & 18 \\
\hline
\end{tabular}

References: (1) Vestrand et al. 2005; (2) Blake et al. 2005 (3) Quimby et al. (2006); (4) Wozniak et al. 2005; (5) Boër et al. 2006; (6) Thöne et al. 2010: (7) Klotz et al. 2008; (8) Gomboc et al. 2008; (9) Perley et al. 2008a; (10) Perley et al. 2008b; (11) Kruehler et al. 2007; (12) Antonelli et al. 2007; (13) Guidorzi et al. 2011; (14) Perley et al. 2011; (15) Chornock et al. 2010; (16) Immler et al. 2010; (17) Gorbovskoy et al. 2011; (18) Cucchiara et al. 2011

cloud. Finally, we apply eq. (7) to fit the rising part of the optical light curve by taking $n_{\mathrm{H}}$ (in the range of $n_{\mathrm{H}}>n_{\mathrm{H}, 0}$ ) and $t_{b}$ as free parameters. The best fit gives us the resulted values of $n_{\mathrm{H}}$ and $\Delta R$.

The resulted values of $n_{\mathrm{H}}$ and $\Delta R$ is also listed in Table 1 Illustrations of our fitting results for the four bursts included in Sample I are shown in Fig.4. From the fitting results, we find that the density of the surrounding molecular clouds are in the range of $10^{3}-10^{4} \mathrm{~cm}^{-3}$, while the size, as implicated by $\Delta R$, is in the order of $\sim 10 \mathrm{pc}$. However, due to small number of GRBs with optical rising part detected in prompt emission, it is impossible to give the statistic discussion of the properties of local molecular clouds. Furthermore, the observed data points in the optical rising part are usually sparse for individual GRB, which may induce large errors in light curve fitting. The future precise observations are needed to test the model and constrain the properties of local environment more precisely.

\subsubsection{Sample II}

For GRBs in Sample II, because there is no optical rising detected due to the delay of optical observations, we cannot well constrain the properties of the surrounding environments. However we still try to make some constraints, although rough. In these bursts, the time $t_{b}$ when the optical-UV flux reaches the plateau, i.e., when the dust destruction front reaches the edge of the cloud, $R_{f}=\Delta R$, can be considered to be smaller than the start time of the optical observations, $t_{\mathrm{op}}$. Thus we have $t_{b}<t_{\mathrm{op}}$. Moreover, we take the flux of the first optical data point to calculate the mean luminosity in optical-UV band. In these bursts, the maximum dust destruction radius must be within the boundary of the cloud, $R_{d}<\Delta R$, otherwise the optical emission from these GRBs cannot emerge. 

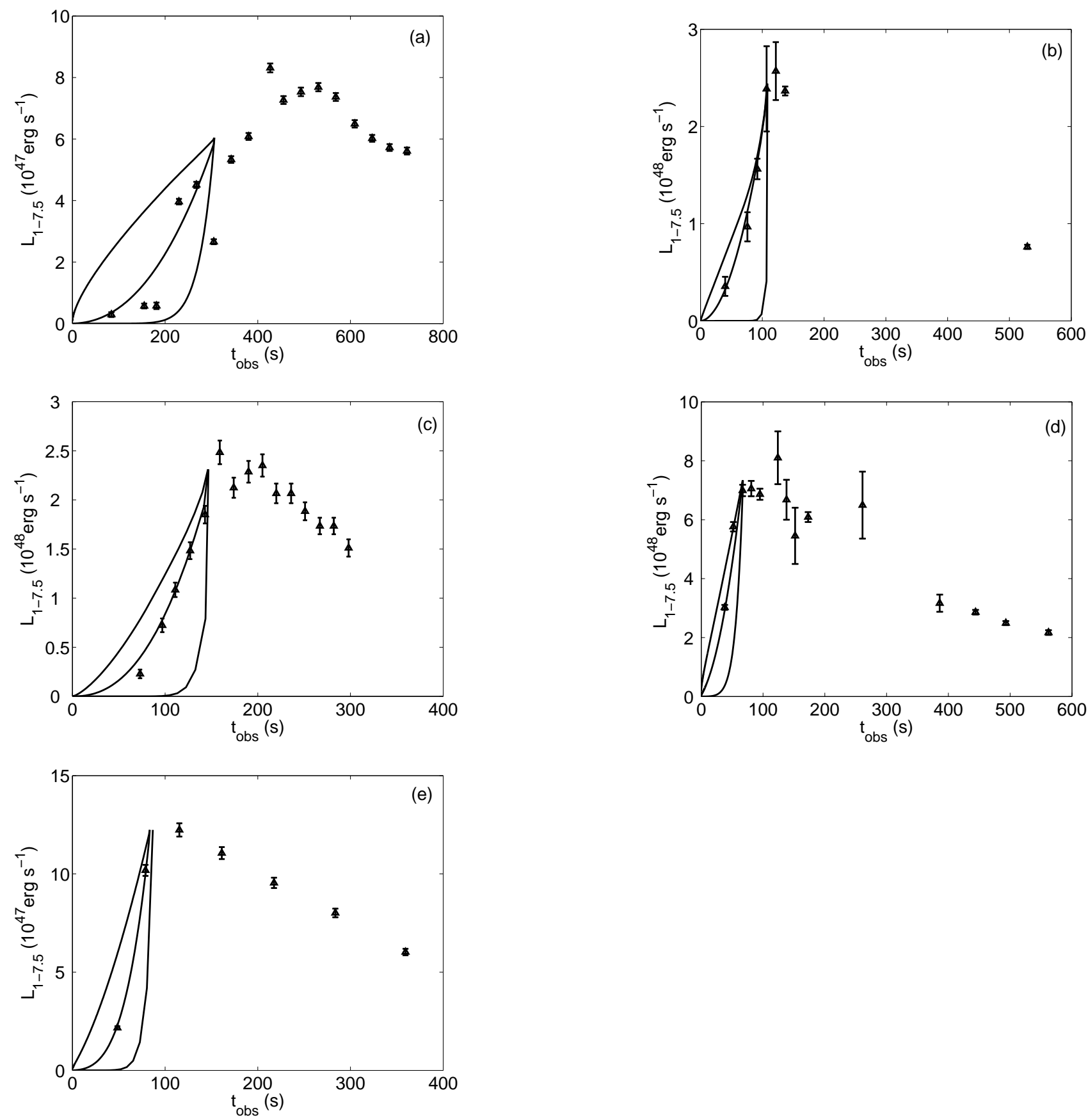

Fig. 4 The light curves of prompt optical emission from four other GRBs in Sample I besides GRB 080319B: GRBs 050820A, 060418, 060607A, 080810 and 100906A. The black triangles are the optical data. The solid lines are the predictions from radiation-dust interaction model, with the parameters $n_{\mathrm{H}}$ and $t_{b}$ marked.

Thus we constrain the properties of the molecular clouds of sample-II GRBs as follows. Considering an optical-UV flash with luminosity $L_{1-7.5}$ and duration of $\Delta t=t_{\mathrm{op}} /(1+z)$, we can calculate the maximal dust destruction radius as function of surrounding density. This puts an upper limit to the value of $\Delta R$ of the relevant GRB. The results for all GRBs in sample II are shown in Fig.5. We see that although there is no good constraints on density $n_{\mathrm{H}}$, the value of $\Delta R$ is quite well constrained since $\Delta R$ does not vary much with $n_{\mathrm{H}}$. All except GRB 041219A have upper limits of $\Delta R<0.1-2$ pc, somewhat less than 


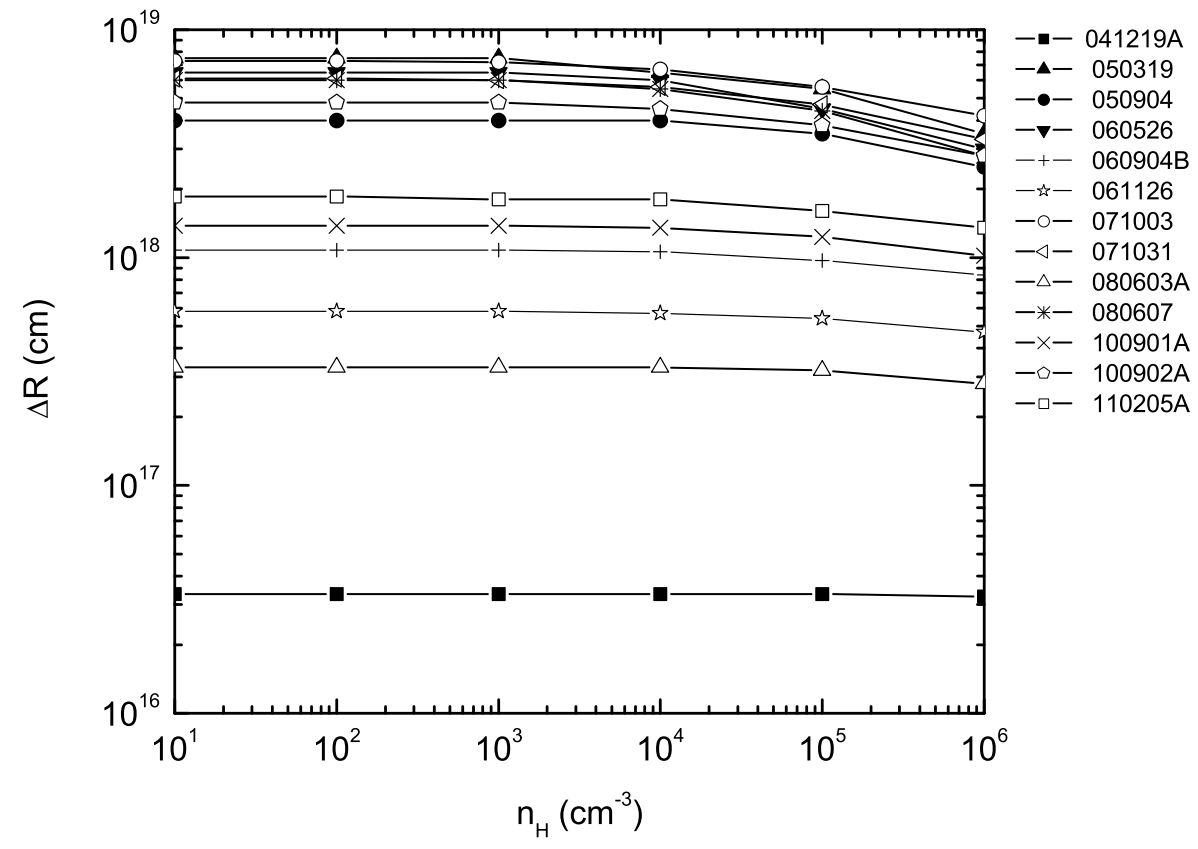

Fig. 5 The constraints on the sizes and densities of molecular clouds around GRBs in Sample II. The allowed parameter region for each GRB is that below the line corresponding to it.

those of sample-I GRBs. This might be reasonable since the luminosity of sample-II GRBs are generally smaller than that of sample-I GRBs. GRB 041219A has exceptionally small luminosity then its value of $\Delta R$ is smaller than $\sim 0.01 \mathrm{pc}$.

\section{DISCUSSION AND CONCLUSIONS}

Long GRBs are believed to be the explosions of massive stars, therefore the GRBs may occur in the molecular clouds where their progenitors were born. We show in this work that the prompt optical-UV emission from GRBs, if originally emitted simultaneously with $\gamma$-ray emission, may appear with relative time delay in observations, due to the dust extinction. This can well explain the optical delayed onset observed in GRB 080319B, and the number density and the size of the molecular cloud are roughly constrained to be $n_{\mathrm{H}} \sim 10^{3} \mathrm{~cm}^{-3}$ and $\Delta R \sim 8 \mathrm{pc}$, respectively. We also investigate the other GRBs with good optical-UV data, and find the densities and sizes of the molecular clouds in the range of $n_{\mathrm{H}} \sim 10^{3}-10^{4} \mathrm{~cm}^{-3}$ and $\Delta R \sim 10 \mathrm{pc}$.

We use a simple picture that the effects of extinction is approximated as a narrowing of the optical pulse, retaining a rectangular profile. This neglects that there may be fluctuation of the original flux with time, and that the dust destruction front is not a zero-thickness one. Thus the constraints on the molecular clouds only make sense by order of magnitude.

The resulted $n_{\mathrm{H}}$ and $\Delta R$ constraints suggest high column densities of gas around GRBs, $\sim 10^{22}-10^{23} \mathrm{~cm}^{-3}$. It is interesting to note that Galama \& Wijers (2001) obtain similar range of column densities by observations of X-ray afterglow spectra. Moreover, our constraints are also consistent with those giant molecular clouds found in Milky Way, which are 
observed to have sizes of $10-30 \mathrm{pc}$ and average gas densities of $10^{2}-10^{3} \mathrm{~cm}^{-3}$ (Winnewisser et al. 1979; Goldsmith 1987). Although the GRB local molecular clouds from our constraints seem to be slightly denser, the low statistics of GRBs with prompt optical detection and the sparse data points for individual GRBs prevent us from giving clear conclusions.

One may expect that the initial fast rise of the prompt optical flux can be produced by the afterglow forward shock due to sweep-up the circumburst medium before deceleration. However, the multi-band observations of two sample-I GRBs 060418 and 060607A show spectral index in the optical band of $F_{\nu} \propto \nu^{-0.9}$ and $F_{\nu} \propto \nu^{-0.8}$, respectively. This implies the injection frequency below the optical band, $\nu_{m}<\nu_{\mathrm{opt}}$, and require extremely unusual afterglow model parameters, e.g., postshock electron energy far smaller than typical value, $\epsilon_{e} \lesssim 10^{-3}$. Moreover, most GRBs in sample I show up-rising even faster than $F_{\nu} \propto t^{3}$ (e.g., GRBs 060418, 060607A, 080319B and 100906A), which is faster than the model prediction for the pre-deceleration forward shock emission at $\nu>\nu_{m}$. One may also expect the up-rising part can be accounted for by the reverse shock emission due to shock swept-up of outflow material but the predicted temporal slope is not faster than $F_{\nu} \propto t^{2}$ (Kobayashi 2000). Thus the prompt optical emission is more likely to be generated within the outflow.

The density of the surrounding from our constraints is higher in general than the medium density implicated by afterglow modeling. The X-ray absorber must lie within $1-5 \mathrm{pc}$ from the GRB thus probing the innermost region in the close vicinity of the GRB explosion. Comparing the HI column densities from Ly $\alpha$ absorption to the metal column densities from X-ray absorption in GRB afterglows, Watson et al. (2007) found there no correlation between the column density values, and the X-ray absorptions often far exceed the HI column densities. Based on a detailed study of the absorption pattern, Campana et al. (2011) found a high-metallicity absorbing medium for GRB 090618 and a best-fitting column densities $6 \times 10^{17} \mathrm{~cm}^{2}$ in Ne and Si. However there is no contradiction here because the size of observed afterglow is usually sub-pc scale, but the region concerned here is in much larger scale, $\sim 10 \mathrm{pc}$, as resulted from the constraints. Thus, it may be that in the place very close to the GRB location the medium density is low while the further-out region has much denser gas. This is reasonable that the vicinity of the GRB source may be affected by the progenitor before the GRB explosion.

In our simple model, for given luminosity and duration of the prompt optical-UV emission, the maximum dust destruction radius can be determined. Once it is within the boundary of the cloud, $R_{d}<\Delta R$, there will be no prompt optical emission observed, neither the optical afterglow emission. The GRB will appear as being optically dark in this case. It is interesting to note that only $60 \%$ GRBs observed by BAT/Swift are detected by UVOT/Swift in the optical afterglows. The "dark burst" (van der Horst et al. 2009) are still mystery now. If dust extinction is the reason, then by our simple model, this suggests that the maximum dust destruction radii and the sizes of the molecular cloud are statistically comparable, i.e., $R_{d} \sim \Delta R$, thus the bright and dark bursts are comparable in numbers. Indeed, as shown in Table 1, those bright GRBs in sample I with better observations and hence better constraints, $R_{d}$ and $\Delta R$ values are similar.

There are quite a few small robotic telescopes that have been built and installed around the world in order to detect the optical counterparts in the early phase of $\gamma$-ray bursts, such as Super-LOTIS (Park et al. 1997), TAROT (Klotz et al. 2009), PROMPT (Reichart et al. 2005), ROTSE-III (RykoR et al. 2009), SkyNe11, WIDGET (Urata et al. 2011), MASTER2, Pi of the sky (Burd et al. 2005), and TORTORA 3 etc. With their large Field of View (FOV) and fast slewing abilities, these telescopes are able to detect the prompt optical emission in minute timescale after the trigger of GRBs by $\gamma$-ray detectors. In the case

\footnotetext{
1 http://skynet.unc.edu/

2 http://observ.pereplet.ru/

3 http://www.eso.org/public/images/eso0808a/
} 
of the "naked-eye GR" 080319B (Racusin et al. 2008), the prompt optical emission was caught by the TORTORA and Pi of the sky even with zero time delay. Besides, the UFFO-Pathfinder (Chen et al. 2011), which aims at prompt optical detection in subsecond timescale, will be launched soon; the Ground-based Wide-Angle Camera array (GWAC), with a larger field of view ( $~ 8000$ square degrees), as a part of ground system of the Chinese-French SVOM mission (Paul et al. 2011), aiming at search for the optical emission in zero delay, will be constructed in the near future. All these robotic telescopes and the planed projects will make a larger and better sample of prompt optical emission from GRBs in the future, leading to more precise constraints on the GRB local environments.

Acknowledgements We would like to thank the useful discussions at pulsar group of PKU and at SVOM group of NAOC. This work is supported by National Basic Research Program of China-973 Program 2009CB824800, China Postdoctoral Science Foundation funded project (No. 20110490590), the National Natural Science Foundation of China (Grant Nos. 111030262) and the Foundation for the Authors of National Excellent Doctoral Dissertations of China.

\section{References}

Akerlof, C. W., et al. 1999, Nature, 398, 400

Antonelli, L. A., Covino, S., D'Avanzo, P., et al. 2007, GCN, 7025, 1

Barthelmy S. D. et al., 2010, GRB Coordinates Network, Circular Service, 11218, 1

Blake, C. H., Bloom, J. S., Starr, D. L., et al. 2005, Nature, 435, 181

Beskin, G., Karpov, S., Bondar, S., et al. 2010, ApJ, 719, L10

Bloom, J. S., Kulkarni, S. R., \& Djorgovski, S. G. 2002, AJ, 123, 111

Boër, M., Atteia, J. L., Damerdji, Y., et al., 2006, ApJ, 638, L71

Burd, A., Cwiok, M., Czyrkowski, H., et al., 2005, New Astronomy, 10, 409

Burenin R. et al., 2008, GCN Circ. 8088

Campana, S. et al. 2001, MNRAS, 410, 1611

Chen, P. et al., 2011, eprint arXiv:1106.3929

Chornock R., Berger E., Fox D., Levan A. J., Tanvir N. R., Wiersema K., 2010, GRB Coordinates Network, Circular Service, 11164,1

Campana, S., Mangano, V., Blustin, A. J., et al. 2006, Nature, 442, 1008

Cucchiara, A., Cenko, S. B., Bloom, J. S., et al. 2011, preprint, arXiv: 1107.3352

de Ugarte Postigo A., Thone C.C., Hjorth J., Jakobsson P., Banhidi Z., Grundahl F., Arentoft T., 2008, GCN Circ. 8089

Draine, B. T. \& Hao, L. 2002, ApJ, 569, 780

Dupree, A. K., Falco, E., Prochaska, J. X., et al. 2006, GCN, 4969

Ferrero, P., Kann, D. A., Zeh, A., et al. 2006, A\&A, 457, 857

Fruchter A. et al., 2006, Nature, 441, 436

Fruchter, A., Krolik, J. H., \& Rhoads, J. E. 2001, ApJ, 563, 597

Galama, T.J., \& Wijers, R. A. M. J. 2001, ApJ, 549, L209

Gomboc, A., Kobayashi, S., Guidorzi, C., et al. 2008, ApJ, 687, 443

Goldsmith, P. F., In: Hollenbach D. J. \& Thronson H. A. eds. Interstellar Processes, Dordrecht: Reidel. 1987, 51

Gorbovskoy, E. S., Lipunova, G. V., et al. 2011, preprint in MNRAS, arXiv:1111.3625

Guhathakurta, P. \& Draine, B. T. 1989, ApJ, 345, 230

Guidorzi, C., Kobayashi, S., Perley, D. A., et al. 2011, preprint in MNRAS, arXiv: 1105.1591

Immler, S. et al., 2010, GRB Coordinates Network, Circular Service, 1159, 1

Kobayashi, S. 2000, ApJ, 545, 807

Klotz, A., Gendre, B., Stratta, G., et al. 2008, A\&A, 483, 847

Klotz A., Boër M., Atteia J. L., Gendre B., 2009, AJ., 137, 4100

Klotz, A., et al. 2011a, GCN 11630

Klotz, A., et al. 2011b, GCN 11632

Kocevski, D., Modjaz, M., Bloom, J. S., et al. 2007, ApJ, 663, 1180 
Kruehler, T., Greiner, J., Afonso, P., et al. 2007, GCN, 7021, 1

Lazzati,D., \& Perna, R. 2002, MNRAS, 330, 383

Ledoux, C., et al. 2005, GRB Coordinates Network, Circular Service,

Ledoux, C., Vreeswijk, P., Smette, A., Jaunsen, A., \& Kaufer, A. 2006, GRB Coordinates Network, Circular Service, 3869

Liang, E. P., Crider, A.,

Markwardt C. B. et al., 2010, GRB Coordinates Network, Circular Service, 11227, 1

Mirabal, N., Halpern, J. P., An, D., et al. 2006, ApJ, 643, L99

Molinari, E., Vergani, S. D., Malesani, D. et al., 2007, A\&A, 469, 13

Paczyński B., 1998, ApJ, 494, L45

Pagani, C. et al., 2008, GCN Rep., 121, 1

Page, K. L., Willingale1, R., Bissaldi, E., et al., 2009, MNRAS, 400, 134

Park H. S., Ables E., Band D. L., et al., 1997, arXiv: 971117

Paul, J., Wei, J., Basa, S. \& Zhang, S. 2011, CRPhy, 12, 298

Perley, D. A., Bloom, J. S., Butler, N. R., et al. 2008a, ApJ, 672, 449

Perley, D. A., Li, W., Chornock, R., et al. 2008b, ApJ, 688, 470

Perley, D. A., Morgan, A. N., Updike, A., et al. 2011, AJ, 141, 36

Perna, R., \& Lazzati, D. 2002, ApJ, 580, 261

Perna, R., \& Raymond, J. 2000, ApJ, 539, 706

Perna, R., Raymond, J., \& Loeb, A. 2000, ApJ, 533, 658

Prochaska, J. X. et al. 2005, GRB Coordinates Network, Circular Service, 3833, 1

Quimby, R. M., Rykoff, E. S., Yost, S. A., et al., 2006, ApJ, 640, 402

Racusin, J. L., Karpov, S. V., Sokolowski, M. et al., 2008, Nature, 455, 183

Reichart, D., Nysewande,r M., et al., 2005, arXiv: 0502429

Rykoff, E. S., Aharonian, F., et al., 2009, ApJ, 702, 489

Sari, R., Piran, T., \& Narayan, R. 1998, ApJ, 497, L17

Sollerman, J., Jaunsen, A. O., Fynbo, J. P. U., et al. 2006, A\&A, 454, 503

Tanvir, N. R., Wiersema, K., Levan, A. J., 2010, GRB Coordinates Network, Circular Service, 11230, 1

Thöne, C. C. Kann, D. A., Jóhannesson, G., et al. 2010, A\&A, 523, 70

Urata, Y., Tashiro, M. S., Tamagawa, T. et al. 2011, PASJ, 63, 137

van der Horst, A. J., Kouveliotou, C., Gehrels, N., et al. 2009, ApJ, 699, 1087

Vestrand, W. T., Wozniak, P. R., Wren, J. A., et al. 2005, Nature, 435, 178

Vestrand, W. T., Wren, J. A., Wozniak, P. R., et al. 2006, Nature, 442, 172

Vreeswijk, P., \& Jaunsen, A. 2006, GCN, 4974

Vreeswijk, P.M., Smette, A., Malesani, D., Fynbo, J. P. U., Milvang-Jensen, B., Jakobsson, P., Jaunsen, A. O., \& Ledoux, C. 2008, GCN Circular 7444

Watson, D. et al. 2007, ApJ, 660, L101

Woźniak, P. R., Vestrand, W. T., Wren, J. A., et al., 2005, ApJ, 627, L13

Waxman, E. \& Draine, B. T. 2000, ApJ, 537, 796

Waxman, E., Mészáros, P., \& Campana, S. 2007, ApJ, 667, 351

Winnewisser, G., Churchwell, E., Walmsley, C. M.In: Chantry G. W. ed.”Modern aspects of microwave spectroscopy”. London, Academic press Inc. 1979, P.313

Woosley S. E. \& Bloom, J. S. 2006, ARA\&A, 44, 507

This manuscript was prepared with the RAA LATEX macro v1.2. 